\title{
The complexity of Rab5 to Rab7 transition guarantees specificity of pathogen subversion mechanisms
}

\author{
Giovanna Mottola ${ }^{1,2}$ * \\ ' UMR MD2, Faculté de Médecine NORD, Aix Marseille University and Institute of Research in Biology of the French Army, Marseille, France \\ ${ }^{2}$ Laboratory of Biochemistry, La Timone University Hospital, Assistance Publique Hôpitaux de Marseille, Marseille, France \\ *Correspondence: giovanna.mottola@univ-amu.fr
}

Edited by:

Benjamin Coiffard, Hôpital Nord, Marseille, France

Reviewed by:

Subramanian Dhandayuthapani, Texas Tech Health Sciences Center, USA

Cecilia Bucci, University of Salento, Italy

Keywords: bacteria, pathogens, Rab5, Rab7, phagosome

Non-pathogenic bacteria are commonly eliminated by the host. Professional phagocytic cells of the immune system, such as macrophages and dendritic cells, recognize and by phagocytosis internalize microbes in specialized endocytic compartments called phagosomes. By fusing with endosomes, phagosomes mature, changing from an early to late state, and early and late endosomes differ in their external and internal biochemical composition. Then, fusion of late phagosomes with lysosomes leads to the formation of an acidic and degradative compartment, the phagolysosome, where bacteria are ultimately eliminated (Fairn and Grinstein, 2012). Bacterial pathogens have evolved several mechanisms to subvert the process of phagosome maturation and to survive and replicate in an intracellular niche that is protected from the immune response. Remarkably, distinct bacterial pathogens can be localized in similar endocytic compartments, suggesting they have a role in the control of the same endocytic steps. The biological machineries controlling endocytosis involve a variety of regulatory events in each step of intracellular membrane trafficking. Here, I would like to summarize and comment on all the discoveries on bacterial pathogens that control the localization or function of the small GTPases Rab5 and Rab7, and therefore modify the maturation from early to late phagosomes, because I believe such a transition is the best way to highlight how bacterial pathogens exploit the complexity of membrane trafficking to establish specific subversion mechanisms.
Phagosome maturation is highly dependent on the endocytic pathway and requires several regulators of this pathway. Rab proteins make up a large family of small GTPases that specifically control various steps in the endosomal and phagosomal transport process (Gutierrez, 2013; Sherwood and Roy, 2013). Each Rab protein is associated with an organelle and a specific step in intracellular trafficking and controls several factors, such as protein and lipid composition of an organelle membrane, fusion between distinct compartments, vesicle motility along microtubules, and interaction with the cytoskeleton. Each compartment has a specific and precise set of Rab proteins, which confers organelle identity. The complexity of Rab protein function is reflected in their life cycle. All Rabs alternate between an active (GTP-bound) state and an inactive (GDP-bound) state. This molecular switch is strictly regulated; one or more guanine nucleotide exchange factors (GEFs) catalyze the release of GDP in exchange for GTP. In the GTP-bound form, Rabs are targeted to their specific organelle membrane by prenylation and recruit a large number of downstream effectors. In the GDP-bound form, they associate with a soluble factor, the guanine dissociation inhibitor (GDI), which stabilizes the inactive species in the cytosol and precludes access to the GEFs. The hydrolysis of GTP to GDP by GTPase-activating proteins (GAPs) terminates the activity of the Rab protein until another activation cycle is initiated.

Rab5 and Rab7 are the bestcharacterized $\mathrm{Rab}$ proteins in the endocytic process and, especially by analogy, in phagosome maturation (Fairn and Grinstein, 2012). Rab5 is mainly associated with early endosomes and early phagosomes and controls the identity and functionality of these compartments. Rab7 defines late endosomes and late phagosomes, and it has been implicated in the transport through these compartments. Therefore, when internalized, bacteria are first localized in Rab5-positive phagosomes and then in Rab7-positive phagosomes. There is much debate about the mechanisms whereby an early Rab5positive endosome or a phagosome becomes a late Rab7-positive endosome or phagosome and whereby Rab5 is consecutively replaced by Rab7 and much effort has been given to understanding the regulation of Rab5 and Rab7 function. The picture so far shows Rabs as complex, highly regulated molecular machineries. A large number of effector proteins interacting with each Rab and of the GEFs and GAPs that regulate their function have been described. In the case of Rab5, for example, more than 60 effectors have been found and several remain to be characterized, and at least 4 GEFs of Rab5 have been characterized so far (Horiuchi et al., 1997; Christoforidis and Zerial, 2000; Kajiho et al., 2003; Otomo et al., 2003; Olchowik and Miaczynska, 2009; Balaji et al., 2012). These discoveries led to a more detailed investigation of the compartment where pathogens reside, which is not simply Rab5- or Rab7-positive, and to a larger comprehension of the molecular mechanisms that bacterial pathogens have evolved. In fact host activities and 
molecules that pathogens modify the Rab5 to Rab7 transition are different and specific for each pathogen (Figure 1). For example, Mycobacterium tuberculosis and Listeria monocytogenes have been localized in modified Rab5-positive endocytic compartment. Nonetheless, at molecular level they distinctly affect the Rab5 machinery. $M$. tuberculosis, responsible for the human disease tuberculosis, enters alveolar macrophages and modifies the formation of phosphatidylinositol 3-phosphate $[\mathrm{PI}(3) \mathrm{P}]$ at the early phagosome membranes (Fratti et al., 2001). The mannose-capped lipoarabinomannan (man-LAM) in the bacterial membrane is released into the phagosomal membrane and inactivates Vps34, the PI(3)P kinase that regenerates $\mathrm{PI}(3) \mathrm{P}$ (Fratti et al., 2003). At the same time, an M. tuberculosis lipid phosphatase, SapM, consumes $\mathrm{PI}(3) \mathrm{P}$ and arrests phagosome maturation (Saikolappan et al., 2012; Puri et al., 2013). PI(3)P is specifically enriched in early endosome/phagosome membranes and stabilizes Rab5 and all its effectors. Its absence in M. tuberculosis infection interferes with the recruitment of the Rab5 effectors and therefore with phagosome maturation to the Rab7 state (Purdy et al., 2005). Interestingly, the M. tuberculosiscontaining phagosome is also enriched for Rab22a, which inhibits Rab7 acquisition and arrests phagosomal maturation (Roberts et al., 2006).

A GEF Rabex5-Rabaptin5 complex is recruited by the active GTP-bound form of Rab5 and through a positive feedback loop regulates Rab5 recruitment to the early endosomes (Lippe et al., 2001). Recent evidence suggests that Rab5 activation is also regulated by RIN1, a RAS effector and a Rab5-GEF (Jiwani et al., 2012; Balaji et al., 2014). Listeria monocytogenes is a gram-positive food-borne pathogen that causes severe infection with symptoms ranging from gastroenteritis to bacterial meningitis and has a mortality rate of about $30 \%$ (Chen et al., 2013). L. monocytogenes invades intestinal epithelial cells and survives in a Rab5positive phagosome until it is prepared to lyse the phagosomal membrane and escape into the cytosol (Farber and Peterkin, 1991). For this purpose, L. monocytogenes acts at the level of Rab5 localization and functions in two steps. First, its attachment to the host cell triggers activation of RIN1, which activates Rab5 for efficient internalization by receptor-mediated phagocytosis and transport to early phagosomes (Jiwani et al., 2012; Balaji et al., 2014). Bacteria then need to block Rab5 activity to avoid maturation of early phagosomes into late phagosomes. Thus, the L. monocytogenes glyceraldehyde-3phosphate dehydrogenase (GAPDH) protein ADP-ribosylates Rab5, rendering this GTPase unresponsive to activation by GEFs, and thereby blocks maturation into Rab7-positive phagosomes (PradaDelgado et al., 2005).

Tropheryma whipplei is a nice example of blockade of endocytic trafficking in an intermediate state of the Rab5 and

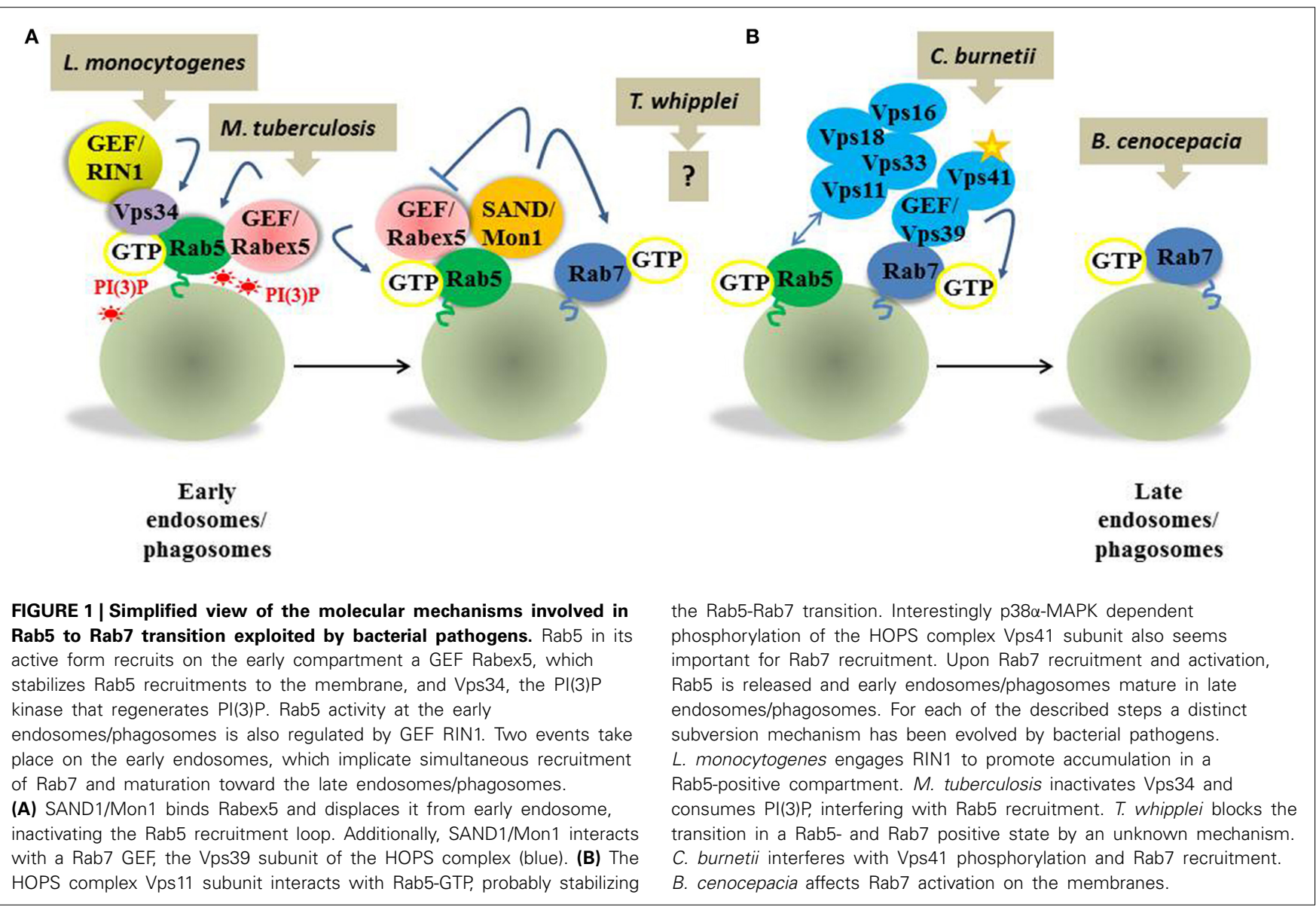


Rab7 transition. This pathogen is responsible for a multi-systemic infection called Whipple's disease, which is fatal without antibiotic treatment (Schneider et al., 2008). T. whipplei resides and replicates in both macrophages and non-microbicidal cells in a phagosome that does not become a phagolysosome (Ghigo et al., 2002, 2010). Recently, the purification and characterization of the intracellular compartment where $T$. whipplei localizes revealed that $T$. whipplei-containing compartments are the first example of Rab5and Rab7-positive phagosomes containing bacteria (Mottola et al., 2014). How is the pathogen establishing this intermediate state? Actually, the transition from a Rab5-positive to Rab7-positive endosome requires both Rabs, and Rab7 is already present on early endosomes together with Rab5 (Poteryaev et al., 2007). Such transition involves two multimeric complexes. The SAND1/Mon1 and ccz1 complex binds Rabex 5 and displaces it from the early endosome, inactivating the Rab5 recruitment loop (Figure 1 and Poteryaev et al., 2007). At the same time, the SAND1/Mon1-Ccz1 complex is also a Rab7 GEF (Nordmann et al., 2010; Cabrera et al., 2014). Intriguingly, Rab5 and Rab7 both bind to the hexameric tethering complex HOPS ("homotypic fusion and protein sorting”). Rab5 binds to subunit Vps11 (Rink et al., 2005). Rab7 interacts with subunits Vps39 and Vps41, but SAND1/Mon1 also interacts with Vps39, which in yeast is a Rab7 GEF (Peralta et al., 2010; Plemel et al., 2011). By a hitherto undescribed mechanism T. whipplei therefore might affect either the function of the SAND1/mon1-ccz1 complex or of the HOPS complex and block endosomes at an intermediate Rab5- and Rab7positive state. Further investigation of the mechanism responsible for the presence of both Rab5 and Rab7 on T. whipplei phagosomes will help us better understand both the T. whipplei infectious process and the regulatory mechanism of the Rab5-toRab7 switch.

The investigation of Coxiella burnetii subversion mechanisms has also revealed insights on pathogen specificity. C. burnetii, the causative agent of the zoonosis Q fever, is also responsible for lethal endocarditis (Raoult et al., 2005). In macrophages, virulent C. burnetii bacteria reside and replicate in compartments known as "phagolysosome-like vacuoles" that have properties of both late endosomes and lysosomes. These compartments do not harbor lysosomal enzymes or Rab7, but they have acidic properties and are positive for lysosomalassociated membrane protein-1 (LAMP-1) (Ghigo et al., 2009, 2012). Recently, by using an avirulent form of this pathogen, Barry A.O. et al. discovered that p38 $\alpha$-MAPK-dependent phosphorylation of HOPS complex Vps41 subunit is crucial for Rab7 recruitment to endosomal membranes (Barry et al., 2012). Remarkably, the lipopolysaccharide of virulent C. burnetii (LPS), a bacterial outer membrane component, is responsible for this subversion mechanism (Barry et al., 2012). Indeed, it interferes with the activation of p $38 \alpha$-MAPK and therefore with Vps41 phosphorylation. Consequently, C. burnetii-containing phagosomes become positive for Rab5, lose Rab5, but do not recruit Rab7.

Burkholderia cenocepacia has evolved a distinct mechanism to interfere with early to late phagosome transition. B. cenocepacia is an opportunistic pathogen that infects patients with cystic fibrosis (Drevinek and Mahenthiralingam, 2010). It can survive within macrophages because it arrests the fusion of phagosomes with lysosomes by acting at the level of Rab7 function (Lamothe et al., 2007; Lamothe and Valvano, 2008). Vacuoles containing B. cenocepacia transiently recruit Rab5 and synthesize PI(3)P. Vacuoles can also acquire the late phagosomal markers CD63 and Rab7, but activation of Rab7 is impaired by the bacteria (Huynh et al., 2010). Findings have indicated that the type III secretion system is not necessary for maturation arrest (Lamothe et al., 2007), and B. cenocepacia also expresses type IV and type VI secretion mechanisms (Aubert et al., 2008; Sajjan et al., 2008), but the identities of the secreted effectors and their mode of action on Rab7 remain unclear.

In conclusion, I have described five distinct pathogens that distinctly exploit the complexity of Rab5 and Rab7 regulation in order to survive and replicate in the host environment. Why is that important? First, this example highlights the coevolution of the two systems. Mammalian cells have evolved a complicated regulation of Rab5 and Rab7 transition in order to guarantee redundancy and therefore "resistance" to any possible dangerous genetic or acquired alteration. Also, pathogens more specifically explore transport steps to establish their own pathogen-specific subversion mechanism. Indeed, such specificity guarantees longer survival and evolution before the host immune system becomes able to find and positively select an adequate immune response. Moreover, this example underlines the strong need for multidisciplinary approaches in the study of infectious diseases. To understand pathogen behavior, membrane trafficking in these pathological contests must be investigated biologically and biochemically. This will be determinant in the development of specific prognostic, diagnostic, and therapeutic tools against an infectious pathology.

\section{REFERENCES}

Aubert, D. F., Flannagan, R. S., and Valvano, M. A. (2008). A novel sensor kinase-response regulator hybrid controls biofilm formation and type VI secretion system activity in Burkholderia cenocepacia. Infect. Immun. 76, 1979-1991. doi: 10.1128/IAI.01338-07

Balaji, K., French, C. T., Miller, J. F., and Colicelli, J. (2014). The RAB5-GEF function of RIN1 regulates multiple steps during Listeria monocytogenes infection. Traffic 15, 1206-1218. doi: 10.1111/tra. 12204

Balaji, K., Mooser, C., Janson, C. M., Bliss, J. M., Hojjat, H., and Colicelli, J. (2012). RIN1 orchestrates the activation of RAB5 GTPases and ABL tyrosine kinases to determine the fate of EGFR. J. Cell Sci. 125, 5887-5896. doi: 10.1242/jcs. 113688

Barry, A. O., Boucherit, N., Mottola, G., Vadovic, P., Trouplin, V., Soubeyran, P., et al. (2012) Impaired stimulation of p38alpha-MAPK/Vps41HOPS by LPS from pathogenic Coxiella burnetii prevents trafficking to microbicidal phagolysosomes. Cell Host Microbe 12, 751-763. doi: 10.1016/j.chom.2012.10.015

Cabrera, M., Nordmann, M., Perz, A., Schmedt, D., Gerondopoulos, A., Barr, F., et al. (2014). The Mon1-Ccz1 GEF activates the Rab7 GTPase Ypt7 via a longin-fold-Rab interface and association with PI3P-positive membranes. J. Cell Sci. 127, 1043-1051. doi: 10.1242/jcs. 140921

Chen, Y., Dennis, S. B., Hartnett, E., Paoli, G., Pouillot, R., Ruthman, T., et al. (2013). FDA-iRISK-a comparative risk assessment system for evaluating and ranking food-hazard pairs: case studies on microbial hazards. J. Food Prot. 76, 376-385. doi: 10.4315/0362-028X.JFP-12-372

Christoforidis, S., and Zerial, M. (2000). Purification and identification of novel Rab effectors using affinity chromatography. Methods 20, 403-410. doi: $10.1006 /$ meth. 2000.0953 
Drevinek, P., and Mahenthiralingam, E. (2010). Burkholderia cenocepacia in cystic fibrosis: epidemiology and molecular mechanisms of virulence. Clin. Microbiol. Infect. 16, 821-830. doi: 10.1111/j.1469-0691.2010.03237.x

Fairn, G. D., and Grinstein, S. (2012). How nascent phagosomes mature to become phagolysosomes. Trends Immunol. 33, 397-405. doi: 10.1016/j.it.2012.03.003

Farber, J. M., and Peterkin, P. I. (1991). Listeria monocytogenes, a food-borne pathogen. Microbiol. Rev. $55,476-511$

Fratti, R. A., Backer, J. M., Gruenberg, J., Corvera, S., and Deretic, V. (2001). Role of phosphatidylinositol 3-kinase and Rab5 effectors in phagosomal biogenesis and mycobacterial phagosome maturation arrest. J. Cell Biol. 154, 631-644. doi: 10.1083/jcb.200106049

Fratti, R. A., Chua, J., Vergne, I., and Deretic, V. (2003). Mycobacterium tuberculosis glycosylated phosphatidylinositol causes phagosome maturation arrest. Proc. Natl. Acad. Sci. U.S.A. 100 5437-5442. doi: 10.1073/pnas.0737613100

Ghigo, E., Barry, A. O., Pretat, L., AL Moussawi, K., Desnues, B., Capo, C.. et al. (2010). IL-16 promotes T. whipplei replication by inhibiting phagosome conversion and modulating macrophage activation. PLOS ONE 5:e13561. doi: 10.1371/journal.pone.0013561

Ghigo, E., Capo, C., Aurouze, M., Tung, C. H., Gorvel, J. P., Raoult, D., et al. (2002). Survival of Tropheryma whipplei, the agent of Whipple's disease, requires phagosome acidification. Infect. Immun. 70, 1501-1506. doi: 10.1128/IAI.70.3.1501-1506.2002

Ghigo, E., Colombo, M. I., and Heinzen, R. A. (2012). The Coxiella burnetii parasitophorous vacuole. Adv. Exp. Med. Biol. 984, 141-169. doi: 10.1007/978-94-007-4315-1_8

Ghigo, E., Pretat, L., Desnues, B., Capo, C., Raoult, D., and Mege, J. L. (2009). Intracellular life of Coxiella burnetii in macrophages. Ann. N.Y. Acad. Sci. 1166, 55-66. doi: 10.1111/j.1749-6632.2009.04515.x

Gutierrez, M. G. (2013). Functional role(s) of phagosomal Rab GTPases. Small GTPases 4, 148-158. doi: $10.4161 /$ sgtp. 25604

Horiuchi, H., Lippe, R., McBride, H. M., Rubino, M., Woodman, P., Stenmark, H., et al. (1997). A novel Rab5 GDP/GTP exchange factor complexed to Rabaptin-5 links nucleotide exchange to effector recruitment and function. Cell 90, 1149-1159. doi: 10.1016/S0092-8674(00)80380-3

Huynh, K. K., Plumb, J. D., Downey, G. P., Valvano, M. A., and Grinstein, S. (2010). Inactivation of macrophage Rab7 by Burkholderia cenocepacia. J. Innate Immun. 2, 522-533. doi: 10.1159/000319864

Jiwani, S., Wang, Y., Dowd, G. C., Gianfelice, A., Pichestapong, P., Gavicherla, B., et al. (2012). Identification of components of the host type IA phosphoinositide 3-kinase pathway that promote internalization of Listeria monocytogenes. Infect. Immun. 80, 1252-1266. doi: 10.1128/IAI.06082-11

Kajiho, H., Saito, K., Tsujita, K., Kontani, K., Araki, Y., Kurosu, H., et al. (2003). RIN3: a novel Rab5 GEF interacting with amphiphysin II involved in the early endocytic pathway. J. Cell Sci. 116, 4159-4168. doi: $10.1242 /$ jcs. 00718

Lamothe, J., Huynh, K. K., Grinstein, S., and Valvano, M. A. (2007). Intracellular survival of Burkholderia cenocepacia in macrophages is associated with a delay in the maturation of bacteriacontaining vacuoles. Cell. Microbiol. 9, 40-53. doi: 10.1111/j.1462-5822.2006.00766.x

Lamothe, J., and Valvano, M. A. (2008). Burkholderia cenocepacia-induced delay of acidification and phagolysosomal fusion in cystic fibrosis transmembrane conductance regulator (CFTR)-defective macrophages. Microbiology 154, 3825-3834. doi: 10.1099/mic.0.2008/023200-0

Lippe, R., Miaczynska, M., Rybin, V., Runge, A., and Zerial, M. (2001). Functional synergy between Rab5 effector Rabaptin-5 and exchange factor Rabex-5 when physically associated in a complex. Mol. Biol. Cell 12, 2219-2228. doi: 10.1091/mbc.12.7.2219

Mottola, G., Boucherit, N., Trouplin, V., Oury Barry, A., Soubeyran, P., Mege, J. L., et al. (2014). Tropheryma whipplei, the agent of Whipple's disease, affects the early to late phagosome transition and survives in a Rab5- and Rab7-positive compartment. PLoS ONE 9:e89367. doi: 10.1371/ journal.pone.0089367

Nordmann, M., Cabrera, M., Perz, A., Brocker, C., Ostrowicz, C., Engelbrecht-Vandre, S., et al. (2010). The Mon1-Ccz1 complex is the GEF of the late endosomal Rab7 homolog Ypt7. Curr. Biol. 20, 1654-1659. doi: 10.1016/j.cub.2010.08.002

Olchowik, M., and Miaczyńska, M. (2009). [Effectors of GTPase Rab5 in endocytosis and signal transduction]. Postepy Biochem. 55, 171-180.

Otomo, A., Hadano, S., Okada, T., Mizumura, H., Kunita, R., Nishijima, H., et al. (2003). ALS2, a novel guanine nucleotide exchange factor for the small GTPase Rab5, is implicated in endosomal dynamics. Hum. Mol. Genet. 12, 1671-1687. doi: 10.1093/hmg/ddg184

Peralta, E. R., Martin, B. C., and Edinger, A. L (2010). Differential effects of TBC1D15 and mammalian Vps39 on Rab7 activation state, lysosomal morphology, and growth factor dependence. J. Biol. Chem. 285, 16814-16821. doi: 10.1074/jbc.M110.111633

Plemel, R. L., Lobingier, B. T., Brett, C. L., Angers, C. G., Nickerson, D. P., Paulsel, A., et al. (2011). Subunit organization and Rab interactions of Vps$\mathrm{C}$ protein complexes that control endolysosomal membrane traffic. Mol. Biol. Cell 22, 1353-1363. doi: 10.1091/mbc.E10-03-0260

Poteryaev, D., Fares, H., Bowerman, B., and Spang, A. (2007). Caenorhabditis elegans SAND-1 is essential for RAB-7 function in endosomal traffic. $E M B O$ J. 26, 301-312. doi: 10.1038/sj.emboj.7601498

Prada-Delgado, A., Carrasco-Marin, E. Pena-Macarro, C., Del Cerro-Vadillo, E., FresnoEscudero, M., Leyva-Cobian, F., et al. (2005). Inhibition of Rab5a exchange activity is a key step for Listeria monocytogenes survival. Traffic 6 , 252-265. doi: 10.1111/j.1600-0854.2005.00265.x

Purdy, G. E., Owens, R. M., Bennett, L., Russell, D. G., and Butcher, B. A. (2005). Kinetics of phosphatidylinositol-3-phosphate acquisition differ between IgG bead-containing phagosomes and Mycobacterium tuberculosis-containing phagosomes. Cell. Microbiol. 7, 1627-1634. doi: 10.1111/j.1462-5822.2005.00580.x

Puri, R. V., Reddy, P. V., and Tyagi, A. K. (2013). Secreted acid phosphatase (SapM) of Mycobacterium tuberculosis is indispensable for arresting phagosomal maturation and growth of the pathogen in guinea pig tissues. PLoS ONE 8:e70514. doi: 10.1371/journal.pone.0070514

Raoult, D., Marrie, T., and Mege, J. (2005). Natural history and pathophysiology of Q fever. Lancet Infect. Dis. 5, 219-226. doi: 10.1016/S14733099(05)70052-9

Rink, J., Ghigo, E., Kalaidzidis, Y., and Zerial, M. (2005). Rab conversion as a mechanism of progression from early to late endosomes. Cell 122, 735-749. doi: 10.1016/j.cell.2005.06.043

Roberts, E. A., Chua, J., Kyei, G. B., and Deretic, V. (2006). Higher order Rab programming in phagolysosome biogenesis. J. Cell Biol. 174, 923-929. doi: 10.1083/jcb.200603026

Saikolappan, S., Estrella, J., Sasindran, S. J., Khan, A., Armitige, L. Y., Jagannath, C., et al. (2012). The fbpA/sapM double knock out strain of Mycobacterium tuberculosis is highly attenuated and immunogenic in macrophages. PLoS ONE 7:e36198. doi: 10.1371/journal.pone.0036198

Sajjan, S. U., Carmody, L. A., Gonzalez, C. F., and Lipuma, J. J. (2008). A type IV secretion system contributes to intracellular survival and replication of Burkholderia cenocepacia. Infect. Immun. 76, 5447-5455. doi: 10.1128/IAI.00451-08

Schneider, T., Moos, V., Loddenkemper, C., Marth, T., Fenollar, F., and Raoult, D. (2008). Whipple's disease: new aspects of pathogenesis and treatment. Lancet Infect. Dis. 8, 179-190. doi: 10.1016/S14733099(08)70042-2

Sherwood, R. K., and Roy, C. R. (2013). A Rabcentric perspective of bacterial pathogen-occupied vacuoles. Cell Host Microbe 14, 256-268. doi: 10.1016/j.chom.2013.08.010

Conflict of Interest Statement: The author declares that the research was conducted in the absence of any commercial or financial relationships that could be construed as a potential conflict of interest.

Received: 20 October 2014; accepted: 05 December 2014; published online: 22 December 2014.

Citation: Mottola G (2014) The complexity of Rab5 to Rab7 transition guarantees specificity of pathogen subversion mechanisms. Front. Cell. Infect. Microbiol. 4:180. doi: 10.3389/fcimb.2014.00180

This article was submitted to the journal Frontiers in Cellular and Infection Microbiology.

Copyright (c) 2014 Mottola. This is an open-access article distributed under the terms of the Creative Commons Attribution License (CC BY). The use, distribution or reproduction in other forums is permitted, provided the original author(s) or licensor are credited and that the original publication in this journal is cited, in accordance with accepted academic practice. No use, distribution or reproduction is permitted which does not comply with these terms. 